\title{
ASPECTOS PSICOJURÍDICOS DA SÍNDROME ALCOÓLICA FETAL
}

\author{
PSYCHOJURIDICAL ASPECTS OF FETAL ALCOHOLIC SYNDROME
}

\author{
Dalila Azevedo da Silva ${ }^{1}$, Eunice Braga Borges dos Santos², Eduardo Jorge Sant'Ana Honorato ${ }^{3}$, \\ Alice Sobral ${ }^{4}$ e Daniel Cerdeira de Sousa ${ }^{5}$ \\ 1 Universidade do Estado do Amazonas, Escola Superior de Ciências da Saúde, Brasil, e-mail: \\ dalilaazevedopsi@gmail.com \\ 2 Universidade do Estado do Amazonas, Escola Superior de Ciências da Saúde, Brasil, e-mail: \\ nicebsantos4@hotmail.com \\ 3 Universidade do Estado do Amazonas, Escola Superior de Ciências da Saúde, Brasil, e-mail: \\ eduhonorato@hotmail.com \\ 4 Universidade do Estado do Amazonas, Escola Superior de Ciências da Sociais, Brasil, e-mail: \\ alicesobral@yahoo.com.br \\ ${ }^{5}$ Universidade Federal de Santa Catarina, PPGPSI, Brasil, e-mail: dancerdeira01@gmail.com
}

\section{ART I CLE INFO}

Article history:

Received 2019-04-25

Accepted 2019-06-17

Available online 2019-06-17
Palavras-chave: Síndrome Alcoólica Fetal. Espectro do Alcoolismo Fetal. Gestação, feto. Alcoolismo.

Keywords:Fetal Alcohol Syndrome. Spectrum of Fetal Alcoholism. Gestation, fetus. Alcoholism.

RESUMO. Este estudo teve como objetivo realizar uma reflexão a cerca dos aspectos psicológicos e jurídicos da Síndrome Alcoólica Fetal - SAF com a intenção de construir um artigo científico. O método adotado foi uma revisão sistemática da literatura de onde se pode depreender que a SAF está se tornando um problema de saúde pública por ser uma das maiores causas de atraso mental. A SAF é uma doença para a qual não existe a cura, porém é totalmente passível de prevenção por meio da abstinência da ingestão de álcool durante o período de gestacional, quando o sistema nervoso está em processo de formação e desenvolvimento. Nesse momento a família surge como fator protetivo, pois a prevenção pode começar em casa junto da família onde as gestantes possam se sentir mais seguras, com a família acompanhando e suprindo as suas necessidades de desenvolvimento das potencialidades. É também, nesse contexto que o psicólogo pode contribuir trabalhando em equipe com os outros profissionais, não só no nível da avaliação como também no nível da intervenção psicológica. Neste estudo não houve definição se a quantidade álcool ingerido pela gestante tem relevância para o feto em desenvolvimento, porém, ficou claro que a completa abstinência dessa substância é de fundamental importância para a prevenção da SAF.

ABSTRACT. This study aimed to carry out a reflection on the psychological and legal aspects of SAF with the intention of building a scientific article. The method used was a systematic review of the literature which can be seen that the SAF is becoming a public health problem because it is a major cause of mental retardation. SAF is a disease for which no cure but is completely preventable by withdrawal of alcohol intake during the gestation period when the nervous system is the formation and development process. At that point the family emerges as a protective factor because prevention can start at home with the family where the pregnant women they can be safer, with family watching and meeting their potentialities development needs is also in this context that the psychologist It presents with with its good practice, serving as a link connection between families and public policies to define strategies of integration and inclusion in society and interaction with other professionals. In this study there was no definition if the amount ingested alcohol by pregnant women is relevant to the developing fetus, but it was clear that the complete withdrawal of this substance is of fundamental importance for the prevention of FAS. 


\section{Introdução}

Conforme a Organização Mundial da Saúde - OMS a dependência alcoólica é considerada como uma das causas que mais acomete a saúde da população com faixa etária entre 15 a 44 anos em todo o mundo, sendo que, parte dessa faixa etária abrange mulheres em idade reprodutiva. Isso acarreta outro problema de saúde pública que corresponde à exposição dos neonatos a sérios riscos, tais como nascimento prematuro, retardo mental, aborto espontâneo, microcefalia, dismorfias craniofaciais, entre outras como a Síndrome Alcoólica Fetal - SAF que acomete o feto a todos os problemas de desenvolvimento e saúde exposto acima. Uma avaliação adequada do problema durante a gestação é fundamental e essencial para o desenvolvimento de um tratamento apropriado assim como na pesquisa de problemas relacionados ao uso nocivo de álcool e da efetividade das intervenções. O SAF é uma entidade nosológica que precisa ser diagnosticada com responsabilidade e que pelo fato de não ser de fácil identificação, na maioria das vezes passa despercebida pelos clínicos em geral. Porém, a prevenção se faz necessária, uma vez que é uma doença evitável e não existe cura, sendo assim, saber como realizar as intervenções com às crianças e suas famílias é de extrema importância para que não se desenvolva, no futuro, para as complicações médicas e de natureza comportamental, emocional e psicopatológica. É nesse contexto que o psicólogo pode contribuir trabalhando em equipe com os outros profissionais, não só no nível da avaliação como também no nível da intervenção psicológica. O objetivo deste estudo foi o de realizar uma revisão do estado da arte a cerca dos aspectos psicológicos e jurídicos da SAF, a fim de construir um artigo científico a ser apresentado à disciplina Saúde Mental, álcool e outras e o Sistema Judiciário. O método adotado foi uma Revisão Sistemática a cerca dos Aspectos Psicológicos e Jurídicos da SAF, nas bases de dados Scielo e BVS - Biblioteca Virtual da Saúde nos últimos 20 anos (1996 a 2015). As buscas mostraram estudos anteriores tendo como foco os aspectos psicológicos e jurídicos da SAF. E como estratégia de busca foram utilizadas as palavras chave: síndrome alcoólica fetal; espectro do alcoolismo fetal; gestação, feto e alcoolismo. Outra estratégia utilizada será a busca reversa (manual) em lista de referência dos artigos selecionados. Foram considerados critérios de inclusão trabalhos publicados em língua portuguesa, publicações Oficiais do Mistério da Saúde, os instrumentos normativos e de políticas públicas além de artigos em sites de referência sobre álcool e outras drogas. 


\section{Desenvolvimento}

\subsection{SÍNDROME ALCÓOLICA FETAL - SAF}

Pinho et al (2006) informa que o termo Síndrome Alcoólica Fetal (SAF) foi descrito por Lemoine e colaboradores pela primeira vez em 1968. Continuando, Ganthous, Rossi e Giacheti (2015) colocam que as alterações físicas e do neuro-desenvolvimento advindas da exposição do feto ao álcool foram complementadas por Jones e Smith em 1973 que estudaram um grupo de crianças inglesas com histórico de exposição ao álcool intra-útero e classificaram uma tríade clínica, formada pelos quadros clínicos: déficit no crescimento, disfunção no Sistema Nervoso Central e características faciais peculiares. Já Clarren e Smith (1978) apontaram o abuso de álcool durante a gestação a causa teratogênica mais frequente da deficiência mental no mundo ocidental, no entanto, só em 1980, é que o "Fetal Alcohol Study Group da Research Society of Alcoholism" estabeleceu os critérios de diagnóstico do SAF. O termo SFA refere-se a uma constelação de anormalidades físicas, comportamentais e cognitivas que ocorrem no feto como resultado do uso de álcool pela mãe durante a gestação.

A SAF de acordo com Lima (2008) se constitui como uma entidade clínica conhecida de muitos anos, porém, pouco reconhecida e identificada por grande parte dos profissionais atuantes na área da saúde pública e da saúde em geral nos dias de hoje e é uma síndrome que se constitui pela ação teratológica do álcool sobre o embrião/feto em decorrência da ingestão de bebidas alcoólicas pela mãe durante a gravidez. E concluem que a SAF é uma condição comum e previsível, porém negligenciada, apesar de o diagnóstico ser relativamente fácil. Conforme Ramalho e Santos (2015), a SAF é uma síndrome conhecida por revelar atraso mental e defeitos físicos em crianças. Ela afeta o crescimento e o desenvolvimento do cérebro causando problemas no sistema nervoso central se estendendo para a memória, o aprendizado, a atenção, a linguagem, o comportamento, além das dificuldades de relação interpessoal o desempenho escolar. Santana et al (2014) corroboram quanto às características da SAF, eles pontuam que as principais são atraso no crescimento pré/pós-natal, baixa estatura, atraso no desenvolvimento, microcefalia, dificuldade na realização de movimentos finos, pode de haver fenda palatina, anomalias articulares e cardíacas e vincos alterados palmares e o dismorfismo facial que pode ser amenizado com o avançar da idade dos sujeitos afetados. Complementando, Lima (2008) salienta que o aspecto clínico da SAF compromete diversos órgãos, afetando diretamente o cérebro, o coração e os rins e apresentam características físicas como face pequena, olhos pequenos e apertados, nariz achatado na base, lábio 
superior fino e com sulco naso-labial, baixa estatura e baixo peso, tais descrições são relatadas por diversos estudiosos e pesquisadores do tema em questão.

2.1. Os efeitos do alcoolismo na gravidez

Desde 1968, estudiosos e pesquisadores tem divulgado as características clássicas, citadas anteriormente, da SAF, porém os maiores efeitos da exposição do feto ao álcool no período pré-natal se da através do cérebro, demonstrando ser este o órgão do corpo do feto em desenvolvimento como o mais afetado no processo de sua formação. As deficiências e danos provocados ao cérebro pela exposição ao álcool no pré-natal são diversos, tais como: deficiência permanente, anomalias neurológicas, disfunções comportamentais, atrasos e deficiência intelectual. As crianças acometidas a SAF também podem apresentar transtornos de comportamento podendo levá-las a dificuldades cognitivas e emocionais (LEMOINE, 1968).

Conforme Pinho et al (2006) os efeitos do álcool consumido na gravidez e no período de amamentação vem desde a antiguidade quando se proibiam os noivos de consumirem bebidas alcoólicas durante a noite de núpcias pois havia o receio de gerarem crianças não saudáveis. Nesse sentido Lima (2008) pontua que na medida em que a gestante bebe o álcool, o mesmo percorre a corrente sanguínea por meio do cordão umbilical e em poucos minutos alcança o sangue do embrião dentro do útero, fazendo com que o feto receba diretamente todo o álcool ingerido pela mãe sem apresentar nenhuma defesa ou proteção da ação destrutiva realizada pela gestante. Nesse sentido Fabbri (2001) considera que a exposição do feto é maior devido ao fato de o seu metabolismo ser mais lento, dessa forma o líquido amniótico permanece com maior concentração de álcool e por mais tempo o que pode gerar a abstinência alcoólica após o nascimento.

Em suas pesquisas sobre abstinência alcoólica Guimarães, Teixeira e Santos (1996) perceberam que esta apresenta características, identificadas nos recém-nascidos por meio de sintomas como agitação, tremores, alteração do sono, convulsões, irritabilidade, hipertonicidade, hiperatividade; nas áreas gastrointestinais apresentaram as alterações de sucção, vômitos, diarreia distensão abdominal; e na área respiratória apareceram os movimentos respiratórios rápidos, depressão, dificuldades respiratórias e por fim as áreas autônomas onde podem se apresentar os Choros de alta tonalidade, espasmos frequentes, febre, alterações cutâneas, bocejos excessivos, rinorreia. Ainda nesse contexto pode-se citar o atraso estaturo-ponderal onde $80 \%$ dos recém-nascidos com a SAF e pouco peso conforme a idade geracional. Cerca de $94 \%$ a $89 \%$ das crianças com a SAF apresentam microcefalia e atraso psicomotor, em relação ao Q.I. quanto mais nítido for o nanismo menor ou mais baixo será o Q.I. da criança. Nesta mesma linha de pesquisa observou-se também que pessoas com a SAF apresentavam problemas com a socialização e comunicação, como 
por exemplo, desajustes comportamentais significativos, impulsividade, comportamento sexual inadequado e menor probabilidade de viverem sozinhas de maneira independente. Essas pessoas podem ainda desenvolver deficiências secundarias que decorrem da exposição e da interação entre as deficiências e os déficits na vida do sujeito (BOAND, 1998).

\subsection{COMO IDENTIFICAR, DIAGNOSTICAR E PREVENIR A SAF}

Conforme o relato de experiências de familiares de portadores da SAF, no nosso país o não existe um exame laboratorial que confirme o diagnóstico, além disso outros distúrbios, principalmente comportamentais, tem características parecidas embora existam pesquisas e estudos realizados fora do Brasil que indicam que há como determinar e quantificar a quantidade de etil éster de ácidos graxos (EEAG), etil glicuromideo (ETG) e etil sulfato (ETS) para avaliar a exposição intrauterina ao álcool por meio de diferentes métodos analíticos. Um deles que pode ser citado como exemplo é o que ocorre por meio da avaliação dos cabelos do neonato refletindo o uso crônico de etanol no sangue no terceiro trimestre de gestação. Outro que também podemos citar como exemplo, é a avaliação do uso de drogas durante o primeiro trimestre de gravidez (JOYA, et al , 2010). Ramalho e Santos (2015) ponderam que apesar dos vários estudos sobre a SAF, ainda não é possível dizer claramente sobre as melhores formas de diagnóstico, avaliação e intervenção devido à dificuldade no diagnóstico e às falhas do mesmo, no nível das áreas envolvendo a criança (família, saúde, escola), muitas vezes estes indivíduos vivem muito tempo sem que sejam corretamente diagnosticados alguns deles apresentarem as mesmas características ou idênticas às de outros transtornos. Conforme nos coloca Lima (2008), no que tange o tratamento da SAF, podem ser incluídos medicamentos para alguns sintomas, terapias comportamental e acolhimento da família a qual a criança se insere, em relação a prevenção a única possibilidade é a abstinência da ingestão de álcool durante o período de gestacional quando o sistema nervoso está em processo de formação e desenvolvimento, sendo assim, a SAF é uma condição previsível e que pode ser evitada.

Já para Ramalho e Santos (2015) a SAF é uma das maiores causas de atraso mental e pode ser prevenido com a abstinência do álcool por parte da gestante, porem isso é de difícil aplicação devido ao fato de as mulheres que usam bebidas alcoólicas nem sempre terem a disposição de parar de beber antes da concepção e muito menos parar depois, além do mais tem a questão de a mulher não perceber a gravidez e não se prevenirem no período pré-conceptual e pré-natal. Dessa maneira faz-se importante o diagnóstico precoce das crianças afetadas pela SAF, assim pode-se tentar diminuir a morbimortalidade e melhorar a sua integração social e proporcionar a essas pessoas condições adequadas seja familiares, educacionais e/ou sociais. Continuando esses autores ponderam que para que isso seja possível é imprescindível que essa intervenção aconteça 
muito cedo, pode começar em casa junto da família onde elas possam se estar mais seguras, com a família acompanhando e suprindo as suas necessidades de desenvolvimento das potencialidades. Nesse contexto o psicólogo se apresenta com o papel de servir como um elo ligação entre a criança e a escola, entre os a criança e os pais a fim de definir estratégias para a boa inclusão na sociedade considerando suas técnicas cognitivas, comportamentais proporcionando o lazer e recreação e também interagindo com a equipe de profissionais e se necessário encaminhando ao neuropediatra para a administração de fármacos para um melhor desenvolvimento da criança.

Para Streissguth (1997) os fatores ou medidas protetores para tais deficiências secundárias podem incluir períodos mais longos em lares estáveis que possam prover carinho e sustento aos mesmos; diagnostico da SAF ou dos efeitos do espectro do álcool fetal (FASD) antes dos seis anos de idade; nunca ter sofrido diretamente com a violência; residência mais longa em cada situação de moradias; experiência com lares de boa qualidade entre as idade de 8 e 12 anos; ter tido o diagnóstico de SAF e ter suas necessidades básicas atendidas durante pelo menos parte de sua vida. Percebe-se por meio dos estudos e pesquisa dos autores que para estimular e proporcionar uma vida mais próxima do possível do "normal" a uma criança com SAF precisa de cuidados imediatos, tais responsabilidades não cabe apenas ao poder público mor meio das políticas públicas de saúde, mas também a sociedade civil em geral por meio de esclarecimentos e cuidados bem como de lares acolhedores. Dessa forma depreende-se que a família tem importância primordial, no sentido de proporcionar os fatores de proteção e qualidade de vida ao sujeito com SAF.

\subsection{A importância da família frente à SAF}

Após o diagnóstico pode ser desenvolvido um plano de intervenção pela equipe multidisciplinar que pode ser composta pelos profissionais da saúde e também da educação a fim de aperfeiçoar o seguimento do paciente. Porém a família e a comunidade precisam estar preparadas e dispostas a participar. Os familiares precisam ser sensibilizados e estar de acordo com a proposta de tratamento/acompanhamento e estar conscientes sobre as consequências psicológicas e sociais do diagnóstico que pode englobar o sentimento de culpa por parte da mãe, da estigmatização da criança para que tenham confiança no processo de diagnóstico e acompanhamento e, tenham inclusive as informações sobre a síndrome, suporte e ajuda comunitária por meio de escolas, hospitais e serviços sociais. Isso envolve vários profissionais conhecedores, tanto do paciente quanto da doença, como professores, médicos, assistentes sociais e psicólogos. É uma proposta que precisa ser acessível e discutido com todas as pessoas que lidam com a criança inclusive com as 
crianças mais velhas, com mais habilidade cognitiva e que precisam obter conhecimentos sobre a sua doença, propiciada pela equipe multiprofissional que atuara como facilitadora/mediadora entre a família e os recursos comunitários no cumprimento de suas recomendações para que a vida dos sujeitos possa ser melhorada e ele tenha qualidade de vida dentro da família e também da comunidade (FREITAS E MESQUITA, 2010).

\subsection{ASPECTOS PSICOJURÍDICOS E SOCIAIS}

Historicamente se tem conhecimento de que o álcool é uma substância que vem acompanhando a humanidade ao longo dos tempos e que seu uso tem sido associado a rituais religiosos, à produção de medicamentos e perfumes e aos momentos das comemorações entre as pessoas. O consumo de bebida alcoólica vem aumentando entre homens e principalmente entre as mulheres na contemporaneidade fazendo com haja um do desenvolvimento de novas técnicas de produção e comercialização desde a revolução industrial a quem coube o papel principal na contribuição para o consumo do álcool pelos trabalhadores que viviam no campo e foram viver na cidade. Conforme os autores abaixo citados esses trabalhadores perderam um pouco da identidade própria, pois houve uma mudança de ritmo em suas e muitas vezes sem a estrutura de apoio da família havendo por parte dos trabalhadores uma tentativa de compensar essa ausência pela sensação de bemestar que o álcool proporcionava (RAMALHO E SANTOS, 2015).

Os pesquisadores Garcia, Rossi e Giacheti (2007), consideram que o consumo de álcool na população aumenta escalonamente, especialmente entre as mulheres. Há um componente ambiental somado aos fatores de pré-disposição genética contribui para o aumento de casos na população e também de recorrência familial condição esta apresentada por este trabalho. Desta forma a SAF está se tornando um problema de saúde pública, embora seja totalmente passível de prevenção, pela não ingestão de álcool durante a gestação As manifestações que constituem o fenótipo desta condição estão presentes ao longo da vida desses sujeitos acarretando-lhes prejuízos sociais e ocupacionais, tornandoos dependentes da assistência da família e do poder público. Os autores entendem que a etiologia da SAF é ambiental e o grau de severidade advém da combinação de ingestão de álcool pela mãe sem deixar de incluir alguns de fatores de risco, tais como quantidade e frequência com que o álcool foi consumido pela gestante, período da gestação em que a mãe ingeriu a substância, idade materna e seus aspectos genéticos, nutricionais e físicos, o uso concomitante de tabaco e/ou outras drogas, fatores socioeconômicos, psicológicos, familiares bem como a resistência da mãe e do feto frente aos possíveis efeitos teratogênicos do álcool. Dessa forma fica subentendido que na falta de políticas públicas de proteção ao feto a principal responsável pela prevenção da SAF é a mãe, já que a completa abstinência ao álcool tanto antes quanto depois da concepção é a única forma de evitar a instalação da SAF. No entanto, existem mecanismos legais que deveriam entrar em 
execução/prática para que essa mãe pudesse evitar o uso de álcool e garantir ao feto, saúde e qualidade e vida ao nascer. Considerando a legislação brasileira aplicada ao uso de álcool, tem-se que não há na jurisdição brasileira leis específicas que disponha sobre o uso de álcool pela gestante. Embora se saiba que o uso de álcool prejudica o feto/embrião em formação, não existe essa preocupação com o bem estar e a qualidade de vida do nascituro. Mesmo porque este pela lei brasileira não é considerado uma pessoa com personalidade mesmo que seja um sujeito de direito. Nesse sentido há uma indefinição quanto a proteção do feto, que de acordo com 0 art. $2^{\circ}$ do Código Civil tem apenas expectativa de direito à vida, caso consiga nascer vivo.

Art. 20 A personalidade civil da pessoa começa do nascimento com vida; mas a lei põe a salvo, desde a concepção, os direitos do nascituro.

Neste sentido pode-se encaixar a questão do tempo da gravidez conforme Fabbri (2001), dependendo do período da gravidez em que o feto esteve exposto ao consumo de álcool pela mãe, ele pode sofrer várias consequências e uma delas é não nascer vivo, não sendo considerado, portanto um sujeito de direitos. Porém são muitos os casos em que o feto consegue sobreviver aos ataques e exposições durante o seu desenvolvimento no ventre materno mas, ele pode nascer afetado pela SAF. E esta, não tem cura, fazendo-se necessário um diagnóstico de responsabilidade a fim de providenciar medidas de promoção da saúde e manutenção da qualidade de vida. Nesse contexto o sujeito com a SAF e sua família ou cuidadores poderão ser beneficiados pelo que preconiza o Art. 227 da Constituição Federal de 1988 que embasou o Estatuto da Criança e do Adolescente que dispõe sobre o melhor interesse da criança e do adolescente em seus arts. $7^{\circ}$ e 11.

Art. 227. É dever da família, da sociedade e do Estado assegurar à criança, ao adolescente e ao jovem, com absoluta prioridade, o direito à vida, à saúde, à alimentação, à educação, ao lazer, à profissionalização, à cultura, à dignidade, ao respeito, à liberdade e à convivência familiar e comunitária, além de colocá-los a salvo de toda forma de negligência, discriminação, exploração, violência, crueldade e opressão. (Redação dada Pela Emenda Constitucional $\mathrm{n}^{\circ} \mathrm{65}$, de 2010)

Art. $7^{\circ} \mathrm{A}$ criança $\mathrm{e} \mathrm{o}$ adolescente têm direito à proteção, à vida $\mathrm{e}$ à saúde, mediante a efetivação de políticas sociais públicas que permitam o nascimento e o desenvolvimento sadio e harmonioso, em condições dignas de existência.

\section{$[\ldots]$}

Art. 11. É assegurado atendimento integral à saúde da criança e do adolescente, por intermédio do Sistema Único de Saúde, garantido o acesso universal e igualitário às ações e serviços para promoção, proteção e recuperação da saúde. (Redação dada pela Lei no 11.185, de 2005)

$\S 1^{\circ}$ A criança e o adolescente portadores de deficiência receberão atendimento especializado. 
$\S 2^{\circ}$ Incumbe ao poder público fornecer gratuitamente àqueles que necessitarem os medicamentos, próteses e outros recursos relativos ao tratamento, habilitação ou reabilitação.

Sendo assim, pode-se dizer que o poder público, juntamente com a sociedade e a família se constitui como mecanismo de proteção da saúde e dos diretos das crianças e adolescentes nesse estado de vulnerabilidade psíquica, jurídica e social. Castro (2014) corrobora dizendo que os direitos postulados pela constituição federal em seu artigo 227 são de aplicação imediata, segundo o art. $5^{\circ} \S^{1^{\circ}}$ da CF/88:

Art. 5ำ Todos são iguais perante a lei, sem distinção de qualquer natureza, garantindo-se aos brasileiros e aos estrangeiros residentes no País a inviolabilidade do direito à vida, à liberdade, à igualdade, à segurança e à propriedade, nos termos seguintes:

$[.$.

$\S 1^{\circ}$ As normas definidoras dos direitos e garantias fundamentais têm aplicação imediata.

O que significa dizer que os direitos fundamentais devem alcançar eficácia máxima e consiste na efetiva execução de tais normas, tratando-se de um direito abrangente, universal e exigível. Porém, mesmo que a constituição estabeleça bases suficientes para a concretização dos direitos ainda existe uma grande lacuna entre a realidade e a lei.

\section{CONSIDERAÇÕES FINAIS}

Desse estudo foi depreendido que a SAF é uma doença que traz vários agravos à saúde do sujeito. É um transtorno que afeta a vida do sujeito em todos os níveis, psíquico, jurídico e social, pois uma vez instalada no feto vai acompanha-lo no seu cotidiano para sempre, pois não tem cura. Embora o diagnóstico da SAF seja difícil em alguns casos, pois requer o reconhecimento dos sinais físicos e clínicos faz-se mister avaliar o histórico positivo de exposição ao álcool intra-útero, bem como o período em que essa exposição se deu. Outro fator que precisa ser levado em consideração e o grau de comprometimento tanto dismórfico quanto comportamental. Neste estudo não houve definição se a quantidade álcool ingerido pela gestante tem relevância, porém ficou claro que a completa abstinência dessa substância é de fundamental importância para a prevenção da SAF. Promoção do bem estar e da qualidade de vida poderá ser garantida por meio de políticas públicas, a participação da família, da escola e de uma equipe multidisciplinar que ficará encarregada de promover a interação entre as esperas públicas e a família.

Com base nas medidas protetivas citadas acima se pode dizer que o poder público juntamente com a sociedade e a família estejam aptos para articularem ações de prevenção da SAF. Seja por meio de um projeto de lei que garanta ao feto o direito de nascer vivo e sadio no sentido de responsabilizar as mães pelo "perigo químico" ao qual estão expondo 
seus filhos, como já é feito em alguns países da América do Norte, seja por meio da uma medida preventiva que dê acesso a informação e conscientização popular entre os jovens em formação. Numa proposta de intervenção com a finalidade de promover conhecimento as jovens que desejam ter filhos e aos futuros pais, pois se sabe que a maioria da população jamais ouviu falar em SAF e nem da sua gravidade. Portanto o direito à saúde, assegurado pela constituição, deve ser garantido mediante políticas sociais e econômicas, dispondo o sistema judiciário de vários instrumentos judiciais e extrajudiciais com a finalidade de atuar junto aos poderes públicos e à sociedade no sentido de prevenção da SAF, um grave problema de saúde publica mundial.

Este trabalho foi apresentado na Semana Nacional de Tecnologia em Itacoatiara, Amazonas, em 2018. Os debates foram bastante produtivos, uma vez que é uma temática pouco abordada na academia, gerando desconhecimento sobretudo sobre os sintomas. Muitos presentes se mostraram surpresos pela existência de tal síndrome e de todos os impactos psicossociais que ela pode trazer, seja para os sujeitos, seja para seus familiares.

\section{REFERÊNCIAS}

BOLAND F.J. Síndrome alcoólica fetal: Implicações para o serviço correcional. Ottawa: Serviço Correcional do Canadá; 1998.

BRASIL. 1988. Constituição da República Federativa do Brasil de 1988. Disponível em http://www.planalto.gov.br/ccivil 03/Constituicao/Constituicao.htm acesso em 12 de novembro de 2015.

. 1990. Estatuto da Criança e do Adolescente. Lei № 8.069, De 13 de Julho de 1990. Disponível em http://www.planalto.gov.br/ccivil 03/Constituicao/Constituicao.htm > acesso em 12 de novembro de 2015.

2001. Código Civil. Lei no 10.406, de 10 de janeiro de 2002. Disponível em http://www.planalto.gov.br/ccivil 03/Constituicao/Constituicao.htm acesso em 12 de novembro de 2015.

CASTRO, L. A. Aplicação da Doutrina da Proteção Integral em situações de vulnerabilidade. Monografia. Escola de Magistratura do Estado do Rio de Janeiro - 2014. Disponível em http://www.emeri.ri.gov.br/paginas/trabalhos conclusao/2semestre2014/trabalhos 22014/Lui zaAlvesdeCastro.pdf> acesso em 08 de novembro de 2015.

FABBRI, C. E., Projeto: Desenvolvimento e validação de um instrumento do uso nocivo de álcool durante a gravidez. Dissertação. Universidade de são Paulo. Faculdade de Medicina de Ribeirão Preto. Depto. de Medicina social. Ribeirão Preto. 2001. Disponível em < http://www.teses.usp.br/teses/disponiveis/17/17139/tde-04092002-092148/pt-br.php> acesso 08 de novembro de 2015. 
FREITAS, M., MESQUITA, M. A. Seguimento de crianças com síndrome alcoólica fetal. In: Efeitos do álcool na gestante, no feto e no recém-nascido/coordenadora Conceição Aparecida de Mattos Segre. Sao Paulo: Sociedade de Pediatria de São Paulo, 2010. Disponível em < http://www.spsp.org.br/downloads/alcool.pdf > acesso em 08 de novembro de 2015.

GANTHOUS. G., ROSSI, N. F., GIACHETI, C. M. Linguagem no Transtorno do Espectro Alcoólico Fetal: uma revisão. Rev. Cefac. 2015, jan-fev; 17(1):253-263, Disponível em $<$ http://www.scielo.br/pdf/rcefac/v17n1/1982-0216-rcefac-17-01-00253.pdf > acesso em 08 de novembro de 2015.

GARCIA R, ROSSI N. F., GIACHETI C. M. Perfil de habilidades de comunicação de dois irmãos com a Síndrome Alcoólica Fetal. Rev. CEFAC vol.9 no.4 São Paulo Oct./Dec. 2007. Disponível em < http://www.scielo.br/pdf/rcefac/v9n4/03.pdf> acesso em 08 de novembro de 2015.

GUIMARÃES H.; TEIXEIRA C.; SANTOS, N. Embriofetopatia alcoólica: Uma experiência. Boletim do Centro Regional de Alcoologia do Porto, 3, nำ11, 1996.

IGLESIAS, F. R. P., Os efeitos do álcool na vida da criança. Monografia de PósGraduação "Lato Sensu”. Projeto A Vez do Mestre. Universidade Candido Mendes. 2004. Disponível em< http://www.avm.edu.br/monopdf/6/FATIMA\%20REGINA\%20PIMENTA\%20IGLESIAS.pdf> acesso em 08 de novembro de 2015.

JOYA, X., PUJADAS, M., FALCÓN, M., CIVIT, E., GARCIA-ALGAR, O., ORIOLALL, O., et al. Cromatografia de massa de gás espectrometria ensaio para a quantificação simultânea de drogas de abuso em placenta humana a 12a semana de gestação. Forensic Sci Int. 2010.

LEMOINE P., HAROUSSEAU H., BORTEYRU J. P., MENUET, J. C., Os filhos de pais alcoólicos anomalias observadas. Cerca de 127 casos. Oeste Med. 1968.

LIMA, J. M. B., Álcool e Gravidez - Síndrome Alcoólica Fetal- SAF: Tabaco e outras drogas. Rio de Janeiro: Editora Científica, 2008.

PINHO, P. J., PINTO, A. L., MONTEIRO, V., Síndrome fetal alcoólico: a perspectiva do psicólogo. Psicologia, saúde \& doenças, 2006, 7 (2), 271-285. Disponível em < http://www.redalyc.org/articulo.oa?id=36270209 > acesso em 09 de novembro de 2015.

RAMALHO, J., SANTOS, M.R. Síndrome Alcoólica Fetal: implicações educativas. Revista Brasileira de educação Especial. Marília. V.21, n. 3, p. 335-344, jul-set, 2015. Disponível em < http://www.scielo.br/pdf/rbee/v21n3/1413-6538-rbee-21-03-00335.pdf> acesso em 08 de novembro de 2015.

SANTANA, R. A., ALMEIDA, L. F. J. L., MONTEIRO, D. L. M. Síndrome alcoólica fetal revisão sistematizada. Disponível em < http://revista.hupe.ueri.br/detalhe artigo.asp?id=501> acesso em 08 de novembro de 2015.

STREISSGUTH, A.P. Deficiências primárias e secundárias na síndrome alcoólica fetal. In: STREISSGUTH, A.P., KANTER J., O desafio da síndrome alcoólica fetal: Superar as deficiências secundárias. Seattle: University of Washington Press; 1997. 